\title{
Emission allocation problems in the maritime logistics chain
}

\author{
Wenting Zhu • Stein Ove Erikstad • \\ Matthias Peter Nowark
}

Received: 13 January 2012/ Accepted: 20 May 2013/Published online: 8 June 2013

(C) Springer-Verlag Berlin Heidelberg and EURO - The Association of European Operational Research Societies 2013

\begin{abstract}
A rational model for emission allocation is a prerequisite for the correct measuring and reporting of the emission footprint in the choice among alternative logistics options. While much attention has been paid to national emission allocations, there has been less focus on cargo-level allocations. In this study, we propose an analytical framework for emission allocation in the maritime logistics chain. This framework provides an entire procedure for the emission allocation of logistics chains with different structures and transported cargoes. A set of universal principles are proposed to form the basis for any rational emission allocation scheme. This includes Completeness, No Redundancy, Simplicity, Fairness, Individual Rationality, Motivation, and Consistency. Three application examples are presented to illustrate this framework: a single logistics chain without return cargo, a single chain with return cargo, and partly uniform cargo with or without return cargo (container shipping). For each of these, different allocation schemes are tested and discussed.
\end{abstract}

Keywords Maritime logistics chains $\cdot \mathrm{CO}_{2}$ emissions - Allocation principles . Allocation schemes

\section{Introduction}

One of the strategies for logistics providers to competitively differentiate themselves in the market is to provide an environmental efficiency message to

W. Zhu $(\bowtie) \cdot$ S. O. Erikstad

Department of Marine Technology, Norwegian University of Science and Technology,

7491 Trondheim, Norway

e-mail: wenting.zhu@ntnu.no

M. P. Nowark

Department of Maritime Transport Systems, Marine Technology Research Institute (MARINTEK), P.O. Box 4125 Valentinlyst, 7450 Trondheim, Norway 
their customers (Olson 2010). A prerequisite for this is the correct calculation and allocation of emissions in the logistic chain. This allocation can provide insight into the responsibility of different players and interested parties with respect to carbon footprint reduction. Furthermore, by allocating the emissions to a specific cargo transport unit, we can achieve the same basis for each transport mode. This makes possible the comparison and benchmarking of emissions in multimodal transport.

For a single-cargo-single-carrier chain, this allocation process is relatively simple. However, current maritime logistics chains are typically more complex, as door-to-door logistics services are increasingly favorable to customers due to road congestion, increasing fuel prices, and increasing concerns about global warming (Vrenken 2005). These maritime chains are usually composed of different transportation modes (e.g., sea/short-sea, road, rail and air), multiple service players (e.g., shipping companies, port authorities, and cargo owners), and multiple cargo types (e.g., general cargoes, containers, four-wheel cargoes, bulk cargoes, liquid cargoes, and passengers). Adding to this complexity, vehicles can be fully or partially loaded with different cargoes with various characteristics, such as chemical carriers, container ships and ferries. Thus, the emission allocation problem is intrinsically complex for maritime logistics services, especially when repositioning moves are involved. It is therefore challenging but necessary to determine a suitable way to allocate the total emissions to similar units for different cargoes and modes (e.g., per container, per pallet, or per ton).

Cargo delivery is the main function of a commercial vehicle. It is therefore reasonable to allocate the emissions from shipping activities to the different types of cargoes. From a logistics chain perspective, this implies that the cargo transported by a vehicle is responsible for all emissions, and that the carrier itself is essentially emission-free. The purpose of an emission allocation scheme is to distribute these emissions to each of the cargoes involved in a chain. This information can be used by cargo owners to determine their products' life cycle carbon footprint.

The remainder of the paper is organized as follows. The literature review provides an overview of the current state-of-research in this area in the second section. This is followed by the proposal of a general framework and principles for emission allocation in the third section, while relevant application examples are described in the fourth section. In the final section, concluding remarks and implications for future work are discussed.

\section{Literature review}

Significant work from different countries and regions has addressed emission allocation at the national level. These describe the allocation of emission allowances or targets across regions or countries based on one or more equity principles (den Elzen et al. 2013). In general, the emissions from all aspects of life should be allocated on the basis of per capita, per unit GDP, or other scaling factors (Sagar 2000; Groenenberg and Blok 2002; Jackson et al. 2006; Chakravarty et al. 2009; Cadarso et al. 2010; Rajagopal and Zilberman 2010; Heitmann and Khalilian 2011). The UNFCCC presented a range of options for the allocation of international bunker 
fuels to nations in its 1996 National Communication by the Subsidiary Body for Scientific and Technological Advice (SBSTA 1996). Here, seven options for allocating emissions to a specific country were proposed, based on (1) the proportion to their national emissions, (2) where the bunker fuel was sold, (3) the nationality of the transporting company, or where the vessel was registered, or the country of the operator, (4) the departure or destination of a vessel, (5) the departure or destination country of cargo or passenger, (6) the nationality of the passenger on flights or the ownerships of cargo, (7) and how much emissions are generated in its national space. However, some of these options have been dismissed later by SBSTA. Based on these options, Heitmann and Khalilian (2011) analyzed the allocation of international shipping emissions by options 1, 2 and 3 . They proposed three indicators to identify a proper scheme: environmental effectiveness, legal effectiveness, and fair burden sharing. Moreover, they compared their results with those based on options 3 and 4 conducted by Faber et al. (2009) and den Elzen et al. (2007) according to these three criteria. They concluded that the option 3, allocating international shipping emissions within a UNFCCC regime on the basis of the operating company should be the best way. This conclusion also implies that it is actually possible and necessary to allocate the emissions on a cargo level.

Although the above-mentioned papers and several published guidelines from different organizations support companies in measuring, reporting and managing their carbon footprints (McKinnon 2010), the main global carbon auditing standards, such as the GHG protocol and ISO16064, do not provide sufficient guidance at the product level in general (McKinnon et al. 2010), or for maritime logistics in particular. This is likely due to the complexity of logistics chains, and/or a lack of sufficient data. McKinnon (2010) discussed the problem with cargo-level carbon audits of supply chains. He suggested that the division of carbon emissions between consignments present an analytical challenge similar to that faced by transport economists (Figliozzi 2006; Houghtalen 2007; Krajewska 2007; Frisk et al. 2010). The latter group has long debated the allocation of common and joint costs between the different consignments on a given vehicle. The allocation rules that companies use are often based on subjective judgments. When a supply chain comprises many separate freight journeys, the cumulative effect of the $\mathrm{CO}_{2}$ allocation decisions made separately for each leg can be substantial. However, no specific rules of emission allocation have yet been presented.

In maritime transport, there has been a significant research focus on ferries, Ro-Ro, and RoPax services. Thus, different allocation schemes have been proposed, and their different allocation methods could lead to substantially different results. In general, the allocation methods, characterized by their applied functional units, can be divided into three types: weight, volume and economic value (Fet et al. 2000; Ziegler and Hansson 2003; LIPASTO 2009; Hagemester and Kristensen 2011).

More relevant to the present work, the MARLEN project proposes an allocation scheme for carbon emissions in a shared network MARLEN (2010). The project team divides the network into three scenarios. The first scenario includes other products and/or products from other product owners in the same mobile unit. In this situation, allocations are made according to the percentage of utilized time and cargo capacity. The cargo capacity here could refer to either weight or volume, 
depending on which of these is the limiting factor. If both are limiting factors, the weight will be used to determine the allocation because weight is a driving factor in emission reduction. The second scenario involves several products and/or products from other product owners in the same terminal. Here, carbon emissions are allocated according to the same principle as those in Scenario 1, taking into account cargo handling equipment and terminal infrastructure. The third scenario includes other parts of the supply chain. No solution is given to this situation due to its complexity. These methods have also been described in (Asbjørnslett et al. 2010). However, no details are provided with regard to making allocations between different transport modes or cargoes. Leonardi et al. 2010 provided a detailed description of allocation methods among multiple customers on the same vehicle. It showed that the loading percentage, based on weight or volume, and traveled distance of different cargoes should be taken into consideration when selecting allocation method. It also suggested that empty running should be required as a part of a journey. However, the empty return issue has not been described in detail in the application example based on a single-leg voyage. Wick et al. (2011) proposed the allocation of GHG emissions from logistics transportation networks based on the Shapley value, which is generally used to solve the cost emission problem. Moreover, Naber (2012) proposed more advanced emission allocation methods. In this study, five different transport network methods are presented: the Star method, the Equal profit method, the Lorenz allocation, the Nucleolus, and the Shapley value. With the exception of the Star method, these methods are all based on cooperative game theory. The time window issue has also been taken into consideration, and four different penalizing methods for allocating extra emissions are discussed. Different allocation schemes are investigated in the specific cases, and the different impacts are presented. It is concluded that the cooperative game theory methods provide a more fair allocation, while the simple method is more robust and easy to implement. In their case, customers must choose a proper method based on their preferences.

The literature review reveals that there are gaps in the current work. First, although there are a number of national emission allocation studies, the purpose of these differs from the purpose of this paper. The papers reviewed here have been more focused on how to allocate the emissions equally in accordance with common but differentiated responsibilities and respective capabilities (UNFCCC 2003). In contrast, the focus of this paper is on emission allocations on the product level. The functional allocation unit will be set as the per cargo unit. Moreover, previous work more specifically related to maritime transportation has primarily focused on ferries and RoPax vessels, representing only a small part of global transport. No comprehensive framework to be used for the maritime logistics chain has been presented yet.

\section{Framework of emission allocation}

\section{General principles}

In this section, we will define certain principles and features that we believe should be aspired for in any rational emission allocation scheme. The first principle is 
Completeness, referring to the allocation of all emissions involved in a chain, including empty return or repositioning legs. The No Redundancy principle is the opposite, stating that a particular emission unit should be allocated once and only once. The third principle is more related to the practical aspects of the allocation process, giving preference to schemes in which data availability and easy accessibility place less burdens on the involved stakeholders (Leonardi et al. 2010). Here, this is referred to as Simplicity. For global emissions, a fair allocation among different countries is a core problem (UNFCCC 2003; Sijm et al. 2001; Heitmann and Khalilian 2011; Naber 2012). For instance, Heitmann and Khalilian (2011) referred to the principle of fair burden sharing to identify a suitable scheme based on the "polluter pays" principle. Similarly, Naber (2012) described fairness to imply that every customer has a fair share of the advantage of joint delivery. We describe Fairness as all emissions should be allocated based on the characteristics of cargoes and the benefits of each cargo owners. The benefits here could be the saving of emissions from coordination or the total finished transport work for each cargo owner, etc. Fairness implies an allocation scheme reflecting to a reasonable degree each cargo unit's contribution to the overall emissions.

Furthermore, we find that some principles are suggested by game theory, particularly by cooperative games, and these are widely used in cost allocation. One of these is individual rationality, which means that no participant pays more as part of a coalition than its stand-alone cost when no coalitions are formed (Frisk et al. 2010). Hence, this principle dictates an upper bound on emission allocation, as players would otherwise have an incentive to switch to other transport solutions, yielding lower emission levels. In this paper, we use Fairness and Individual Rationality together to describe a reasonable allocation. The principle of Fairness can in some cases be contrary to that of Individual Rationality, where each stakeholders bargaining position may be decisive. The level of fairness will to some extent be a subjective measure reflecting how the different stakeholders perceive a given scheme. The principle of Motivation refers to the efficiency of the scheme to provide correct incentives to emission reduction.

Table 1 Requirements and features of emission allocation

\begin{tabular}{|c|c|}
\hline \multicolumn{2}{|c|}{ Description of general principles } \\
\hline Completeness & All emissions, including empty return trips, should be accounted for and allocated \\
\hline No Redundancy & The same emissions should be allocated only once, thus avoiding double-counting \\
\hline Simplicity & $\begin{array}{l}\text { The required data for a scheme should be easily available, and the calculation } \\
\text { method should be easily understandable, effectively implementable and as simple } \\
\text { as possible }\end{array}$ \\
\hline Fairness & $\begin{array}{l}\text { The emissions should be allocated based on the characteristics of cargoes, reflecting } \\
\text { to the degree possible each cargo unit's contribution to the overall emissions }\end{array}$ \\
\hline $\begin{array}{l}\text { Individual } \\
\text { Rationality }\end{array}$ & $\begin{array}{l}\text { No cargo should be allocated more emissions than it would have if no other cargoes } \\
\text { were involved in the chain }\end{array}$ \\
\hline Motivation & $\begin{array}{l}\text { The scheme should provide insights into opportunities and incentives for improving } \\
\text { environmental efficiency }\end{array}$ \\
\hline Consistency & $\begin{array}{l}\text { The functional unit should be consistent across transport modes to be applicable for } \\
\text { benchmarking multimodal logistics chains }\end{array}$ \\
\hline
\end{tabular}


Furthermore, as we mentioned above, the emission allocation also can serve for the benchmarking across multiple transport modes, the Consistency in the approaches used for other transport modes should be considered (Wood et al. 2008; Leonardi et al. 2010). Overall, a meaningful allocation scheme should therefore satisfy the principles described in Table 1.

No scheme will meet all of the principles listed above. For instance, the search for a motivated approach may bring solutions that are not simple or easily understandable. Additionally, there may be a trade-off between Simplicity and Consistency, as the latter requirement is likely to imply relatively more complexity when more transport modes are included. Therefore, we need to find a reasonable trade-off between these principles. However, requirements such as Completeness and No Redundancy must be met for the approaches used in this paper. Other principles could depend on the customer's applicability.

\section{Analysis framework of emission allocation}

In this section, a general procedure for emission allocation is proposed. For the development of the allocation schemes, we can start with a port-to-port operation, which is typically a basic element in a maritime logistics chain. Emission allocation for a complex logistics chain can be determined by combining these simple legs. For instance, an entire intermodal supply chain is a combination of each different leg with a specific type of vehicle. Therefore, it is possible to use combinations of different scenarios in this study to discuss the emission allocation issues of an intermodal logistics chain. All major notations used are listed below in Table 2. The

Table 2 Notations used in the models

Notations and descriptions

Sets

$N \quad$ Set of logistics nodes indexed by $i$

$M \quad$ Set of cargo indexed by $m$

A Set of logistics legs $i j$

Parameters

$E \quad$ The total emissions from a complete roundtrip (tons)

$E_{i j}^{\mathrm{T}} \quad$ Emissions from transportation from port $i$ to $j$ (tons)

$E_{i}^{\mathrm{H}} \quad$ Emissions from handling activities at port $i$ (tons)

$E_{i}^{\mathrm{W}} \quad$ Emissions from waiting in port $i$ (tons)

$A_{m} \quad$ Subset of arcs of a voyage for which a cargo $m$ is on board

$Q_{m i j} \quad$ Total transport units of cargo $m$ on leg $i j$ (tons, $m^{3}$, lane meters, number of container, number of passenger, or number of cars, etc.)

$e_{m} \quad$ Unit emission of cargo $m$ (tons/transport work unit)

$D_{i j} \quad$ Transport distance between two logistics nodes $i$ and $j(\mathrm{~km})$

$\gamma_{m}$ Emission allocation factor for each cargo $m$, which depends on different cargo characteristics and allocation schemes

$P_{m} \quad$ Distribution of emission relevant to different allocation methods of each cargo $m(\%)$ 


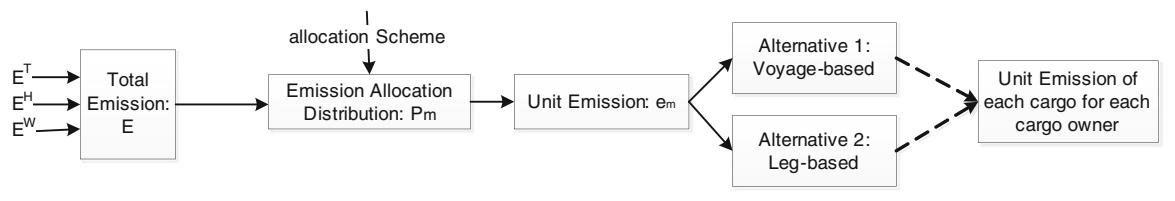

Fig. 1 General emission allocation framework

framework (see Fig. 1) proposed here includes the basic process of the emission allocation, which can be divided into four steps as follows. We will provide detailed explanations below.

- Calculation of total carbon emission

- Analysis of different allocation schemes

- Calculation of the emission distribution $P_{m}$

- Calculation of unit emission for each type of cargo $e_{m}$.

\section{Step 1: Calculation of total carbon emission}

For this first step, the total emissions can be calculated based on the fuel consumption during transportation (from one point to another), waiting, and in-port cargo handling. Because the emissions are fuel-based, we use the bottom-up method to calculate the total carbon footprint. We use an "activity-based bottom-up" approach to estimate fuel consumption for individual categories of ships. The estimates of fuel consumption are then added together to find the global total (IMO 2000). Thus, the total emissions from a port-to-port chain are given by:

$$
E=\sum_{(i, j) \in A} E_{i j}^{\mathrm{T}}+\sum_{i \in N} E_{i}^{\mathrm{H}}+\sum_{i \in N} E_{i}^{\mathrm{W}}
$$

Step 2: Analysis of different allocation schemes

We divide the cargoes into three classes:

- Weight-sensitive cargo: cargoes for which weight is the limiting factor for the vessel; these include coal and ore.

- Volume-sensitive cargo: cargoes for which volume is the limiting factor for the vessel; these include industrial parts, furniture, and clothes. These also can include such cargoes as containers and cars.

- Value-sensitive cargo: cargoes with a high value-to-weight ratio in transport, such as medical equipment and urgent delivery items.

In principle, the existence of these different cargo types requires different allocation methods: the weight-based approach, the volume-based approach, and the economic value-based approach. In particular, the value-based method could be used for the value-sensitive cargoes mentioned above or for a mixture of different types of cargo, for instance, the ferry transport. In this case, it is sometimes difficult to find a measurement standard, such as weight or volume, for all of the cargoes and passengers. Under such circumstances, the value-based approach could be an option. The economic value could be the real value of the cargo itself, the freight rate, or the ticket price of the cargo, depending on the different transport modes 
involved. For instance, the economic turnover generated by each passenger and cargo is used as a basis for emission allocation (Fet et al. 2000). Moreover, when using the value-based method, those approaches used in cost allocation could also be introduced for emission allocation, for instance, Shapley value, which is one of the most popular methods for cost allocation. Using Shapley value, the allocation can be based on the marginal contribution of emission savings of each cargo type. This gives each player their average contribution when joining up in a transport work (Naber 2012). However, most of the methods used in cost allocation are more complicated and advanced. The complexity and workload of calculation will increase when the number of players in a transport network increases.

We can divide this allocation problem into different scenarios based on the complexity of the maritime logistics chains:

- Scenario 1: A single cargo-type vessel without return goods (Simple base example): In this scenario, only one type of cargo is carried. It is therefore not difficult to select a method according to the characteristics of this cargo.

- Scenario 2: A single cargo-type vessel with return goods (Extension example 1): In maritime transport, the one-ship-one-cargo situation is very common. Here, one cargo refers to the same type of cargo. The main difference in this extension is that we allocate the emission based on either the complete roundtrip or separated legs.

- Scenario 3: Partly uniform cargoes (Extension example 2): We investigate allocation schemes for cargo that is uniform in some dimensions but different in others. One typical example is containers with a standard shape and volume but with different weights and values since the cargoes inside each container have different characteristics. We propose a simplified approach in this paper that facilitates the emission allocation in such complex circumstances.

- Scenario 4: Multiple types of cargo with or without return goods: Ferry transport is a typical case for this scenario, as it is a mix of wheel-based cargoes, cars and passengers.

- Scenario 5: Repositioning: In the context of our study, repositioning refers to the situation in which a vessel is required to transport some goods from one point to another. Instead of going back directly afterward, the vessel sails empty to the third point to pick up other cargoes from the required destination. This could be from the original point or a different point. If it is a new point, the vessel returns empty to its point of origin after unloading. According to the allocation principles proposed above, we know that all of the emissions from empty legs should be allocated to the transported cargoes. Therefore, the question arising in this scenario is how to fairly allocate the emissions from the empty legs. The Completeness principle dictates that the emissions used for repositioning must be carried by the cargo owners. However, they might not be willing to do so because an empty return trip generates lower costs and emissions. If different types of cargoes are involved, this further increases the complexity of the situation. In such cases, game theory can be used to provide an upper bound.

We will not present more detailed discussion for last two scenarios in this paper. For Scenario 4, relevant discussion can be found in the reference provided above, while more discussion on Scenario 5 will be a part of our future work. 


\section{Step 3: Calculation of the emission distribution $P_{m}$}

Based on the analysis of allocation schemes, we can obtain the emission distribution factor $P_{m}$, which indicates how much emissions should be allocated to each type of cargo of the total emissions in a roundtrip. It means that the allocation scheme used in this paper is a proportional allocation approach, the point of which is to find a reasonable and accepted proportion, namely $P_{m}$ for each cargo. $P_{m}$ can be expressed by the following formula based on the transport work of each cargo type:

$$
P_{m}=\frac{\sum_{(i, j) \in A_{m}} \gamma_{m}\left(Q_{m i j} D_{i j}\right)}{\sum_{m \in M} \sum_{(i, j) \in A_{m}} \gamma_{m}\left(Q_{m i j} D_{i j}\right)}
$$

where,

$$
\sum_{m \in M} P_{m}=1
$$

It can be noticed that for a single leg, the value of $D_{i j}$ can be removed from the expression above. Moreover, if the quantity of a cargo does not change during the voyage, we can simply use $Q_{m}$ instead of $Q_{m i j}$.

The value of the emission allocation factor $\gamma_{m}$ can be understood as a coefficient given to a type of cargo based on the characteristics of cargo itself and allocation schemes. It could be different for the same type of cargo if the chosen schemes are different. For instance, if the emissions are allocated by weight-based approach, the value of this allocation factor given for heavy cargoes should be higher than that for the light ones. For the first two scenarios, the emission allocation factor is independent of allocation scheme:

- For Scenario 1, we can set that $\gamma_{m}=1$.

- For Scenario 2, we have $\gamma_{1}=\gamma_{2}$. This is because the types of cargo on the forward trip and return trip are the same. In fact, we can set that the same value for the same type of cargo.

For the other aforementioned scenarios, this value depends more on different allocation schemes:

- For the partly uniform cargo described in this paper, as in Scenario 3 for container shipping, $Q_{m i j}$ is the number of TEU. According to the allocation schemes, $\gamma_{m}$ could be decided based on the average weight per TEU, the size of different containers, or the freight rate per TEU. More discussion will be given in an application example in the next section.

- In cases of multiple types of cargo, such as passengers and cars on a ferry carrier, $Q_{m i j}$ is the number of passengers or cars. The allocation factor $\gamma_{m}$ can be decided based on the average weight of each passenger or car, the total capacity saved for passengers and cars (Hagemester and Kristensen 2011), or the economic turnover on the basis of ticket price for each passenger and car (Fet et al. 2000). For instance, Sandvik (2005) used a weight-based method to allocate emissions for a ferry transport. Passenger vehicle unit (PBE) was proposed as a transformed unit, which passengers and freight can be converted 
into. In their study, one PBE equals three transport units. Accordingly, one passenger is considered to be $1 / 3 \mathrm{PBE}$ or one transport unit, while one HGV counts as 5 PBE or 15 transport units. A similar way of defining value of this factor for ferry transport can be found in Hagemester and Kristensen (2011).

Step 4: Calculation of unit emission for each type of cargo $e_{m}$

In this step, we allocate the total emissions to the different cargoes to obtain the unit emission. The unit emission refers to emissions generated from the transportation of goods or passengers by a specific means of transport and can be expressed as emissions per ton-kilometer, per passenger-kilometer, or per containerkilometer. Such information is necessary and helpful for companies who want to compare emissions across multimodal logistics chains and to record and track their performance over time. It also allows them to perform benchmarking against competitors (Leonardi et al. 2010).

There are two ways of obtaining unit emission. One is voyage-based, and the other is leg-based, which can be regarded as a special case of voyage-based method. The unit emission according to a voyage-based method is given by:

$$
e_{m}=\frac{E P_{m}}{\sum_{(i, j) \in A_{m}} Q_{m i j} D_{i j}}, \quad m \in M
$$

The alternative, leg-based method, is useful, for instance, when the cargoes with the same type belong to different cargo owners or have different transport requirements in terms of delivery destination, or schedule. It can be expressed as:

$$
\left(e_{m}\right)_{i j}=\frac{E_{i j} P_{m}}{Q_{m i j} D_{i j}}, \quad m \in M,(i, j) \in A_{m}, i, j \in N
$$

Here $E_{i j}$ refers to total emissions on leg $i j$.

\section{Application examples}

In this section, we give some examples of emission allocation applications for the different scenarios mentioned above: a single cargo-type vessel without return goods, a single cargo-type vessel with return goods, and partly uniform cargoes (container shipping) (see Fig. 2). The first two examples constitute the most basic logistics chain; hence, a complex chain could be a combination of these scenarios. In the third example, the discussion could provide insight into the intermodal logistics chain, as containers are the most common transport unit of an intermodal logistics chain.

In all three cases, we assume there are two ports, namely port $\mathrm{A}$ and port $\mathrm{B}$, which means that $i, j \in N=\{A, B\},(i, j) \in A_{m}=\{A B, B A\}, m \in M$. For the first two examples, we also assume that the quantity of this single cargo in a voyage does not change, therefore, $Q_{m}$ can be used directly. A brief summary of the illustrated cases is presented in Fig. 2. As discussed in terms of the four-step process, Fig. 3 provides an outline of these three scenarios. 


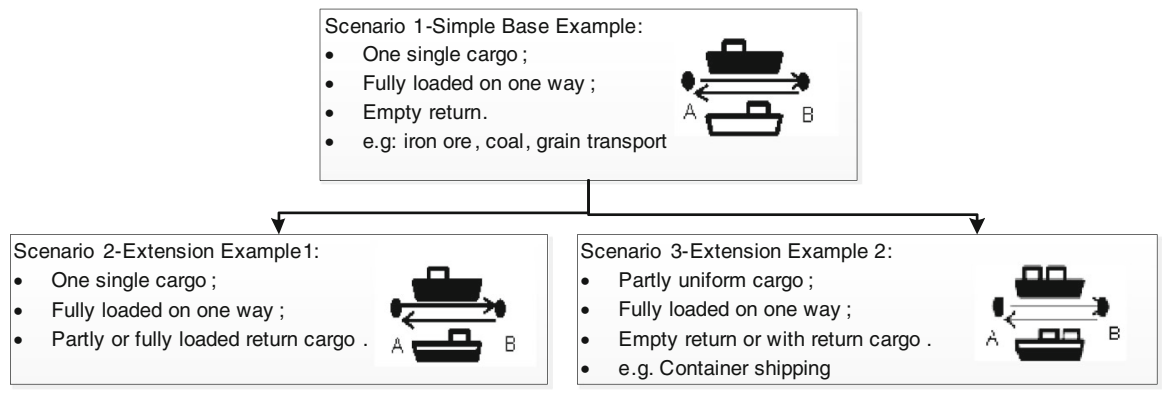

Fig. 2 Outline of simple application examples

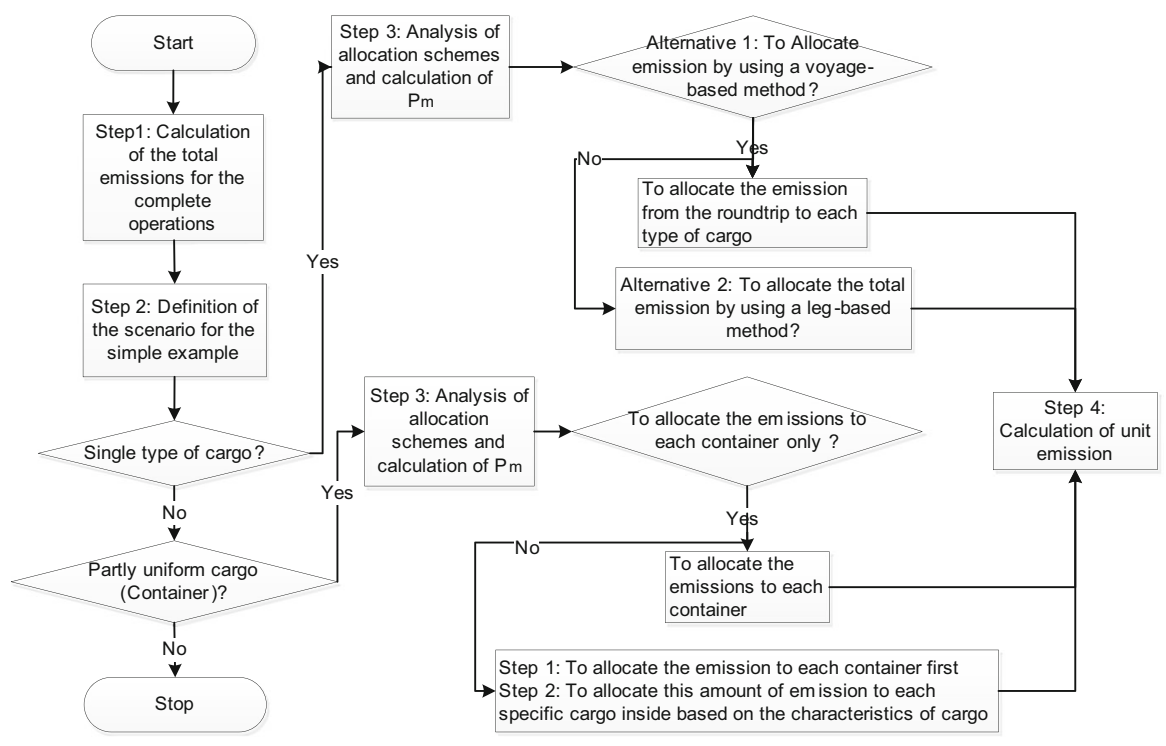

Fig. 3 Emission allocation process for the illustrated examples

Single cargo with empty return

In this simple base case, we assume that only one type of cargo is transported from port A to port B by the vessel. Because there is only one cargo type involved in this base case, the emission allocation will be directly related to its characteristics. The main question in this case is whether the cargo in the forward leg should also take responsibility for the emissions generated during the empty return. According to the Completeness and No Redundancy principles discussed above, all of the emissions from a complete trip, including the empty return, are allocated to the transported cargo. Therefore, for this scenario, the voyage-based allocation method can be applied, that is the unit emissions are actually the average emissions from the roundtrip. We first can obtain $P_{m}=1$ for all potential methods. Then, the unit emission for this cargo type $m$ is: 


$$
e_{m}=\frac{E_{A B}+E_{B A}}{Q_{m} D_{A B}}, \quad m \in M
$$

For illustration, we take iron ore transportation as an example. This usually involves full shiploads and an empty return. We assume that $E_{i j}=E_{j i}=E, Q_{1}=$ $Q, D_{i j}=D_{j i}=D, i, j \in N=\{A, B\},(i, j) \in A_{1}=\{A B, B A\}$. We allocate the emissions based on weight because iron ore (cargo 1) is a type of weight-sensitive commodity, and we obtain the unit emission for this roundtrip:

$$
e_{1}=\frac{E_{A B}+E_{B A}}{Q_{1} D_{A B}}=\frac{2 E}{Q D}=2 e
$$

Here, to make it simple to compare unit emissions across alternative allocation schemes, we define $\frac{E}{Q D}=\mathrm{e}$.

Single cargo-type vessel with return goods

Scenario 2 is an extension of Scenario 1. In general, the types of cargo on the forward and return routes are the same, as in "one ship one cargo" transport. This means that the same method can be applied to both legs, which helps simplify the allocation process. For the first alternative, voyage-based method, the unit emission can be expressed as follows:

$$
e_{1}=\frac{E P_{1}}{Q_{1} D_{A B}}, \quad e_{2}=\frac{E P_{2}}{Q_{2} D_{B A}}
$$

where

$$
P_{1}=\frac{Q_{1} D_{A B}}{Q_{1} D_{A B}+Q_{2} D_{B A}}, \quad P_{2}=\frac{Q_{2} D_{B A}}{Q_{1} D_{A B}+Q_{2} D_{B A}}
$$

with $\gamma_{1}=\gamma_{2}$.

The alternative is the leg-based method, by which the unit emissions of the forward trip and the return trip are calculated individually:

$$
\begin{aligned}
& \left(e_{1}\right)_{A B}=\frac{E_{A B}}{Q_{1} D_{A B}} \\
& \left(e_{2}\right)_{B A}=\frac{E_{B A}}{Q_{2} D_{B A}}
\end{aligned}
$$

where $P_{1}=P_{2}=1$.

We use the same example for the one without return goods above for illustration. Now, we assume that the shipping company does find additional cargo for the return trip, which is also a type of heavy goods with a weight of $1 / 2 Q$. The amount of emissions from this return trip $E_{B A}$ is assumed to be $E$ as above: $\gamma_{1}=\gamma_{2}, P_{1}=2 /$ $3, P_{2}=1 / 3$.

$$
e_{1}=\frac{2 E P_{1}}{Q D}=\frac{4}{3} e
$$




$$
e_{2}=\frac{2 E P_{2}}{\frac{1}{2} Q D}=\frac{4}{3} e
$$

We also can allocate the emission based on the second alternative, according to Eqs. (10) and (11) above, we get the unit emission for each cargo on each leg:

$$
\begin{gathered}
e_{1}=\frac{E}{Q D}=e \\
e_{2}=\frac{E}{\frac{1}{2} Q D}=2 e
\end{gathered}
$$

If we compare the results from these two methods, we can find there is an apparent difference because of the utilization of two legs. If the utilization factor was $100 \%$ on both the forward and return leg, they would give the same result for both the forward and return cargo owner. However, if the return cargo utilization factor is $<100 \%$, they will differ. Moreover, both methods are easy to implement and adhere to the basic principles such as Completeness, No Redundancy, and Individual Rationality. In terms of Motivation principle, it will play differently depending on the stakeholders' perspective. Both schemes motivate the forward cargo owner to seek return cargo, but he will be better off with the leg-based allocation. However, for the system as such, the voyage-based allocation will be best with respect to Motivation, since both actors will gain from an increased utilization rate from the other part.

\section{Partly uniform cargoes}

In this section, we discuss the third scenario and use container shipping as an example of a partly uniform cargo type. Containers are a type of package with standard sizes (i.e., 20- and 40-ft containers) that are considered uniform. In contrast, cargoes inside a container could have different characteristics and they could be weight-sensitive, volume-sensitive, or value-sensitive in nature. Thus, they are considered partly uniform. As a consequence, the emission allocation has two steps: first, the emissions are allocated to each container, and second, they are further allocated to the different cargoes inside the containers. The return cargo is another key issue to be considered in container shipping. This case is of practical significance in the maritime logistics chain and deserves careful attention. One reason is that container shipping represents a major mode of maritime transportation in terms of both geography and trade volume. In general, return trip utilization could account for 20-70\% of the total DWT capacity (Asbjørnslett et al. 2010). Under such circumstances, if we can increase the utilization of those return trips, we have the potential to decrease the unit emission of container shipping and thereby improve the environmental efficiency.

We start with a simple example first. We assume that there are several different cargoes in a certain number of containers that need to be delivered from port A to port B by a container vessel. These cargoes have different characteristics in terms of weight and monetary value, but all are transported in $20 \mathrm{ft}$ containers. We consider four different types of containers: light and heavy containers of high value and light 
and heavy containers of low value (see Table 3). First, we calculate the total emissions from transport by this vessel according to input parameters, such as fuel type, daily fuel consumption, distance of the chain, and vessel speed (Kontovas and Psaraftis 2011). Then, different methods can be used to allocate the emissions on the basis of container characteristics (volume or size, weight and value), and other factors such as quantity. The value of $\gamma_{m}$ differs based on the volume, unit weight, and unit value of each type of container. In this case, since we assume all the containers are $20 \mathrm{ft}$, the value of $\gamma_{m}$ is the same for all types of containers when a volume-based method is used. But this value is varying across different types of container when a weight-based or value-based method is used. Accordingly, emission distribution $P_{m}$ can be obtained (see Fig. 4). More detailed discussion on the selection of these two factors is given in the following part of this section.

From Fig. 4, we find considerable differences between these three methods of emission allocation in container shipping. This means that the final selection of the approach has a significant impact on the emission footprint of different cargoes. Moreover, if the cargoes belong to different owners, these owners might have distinct preferences with respect to their own interests. Thus, several questions arise here: Which method should be selected to make a sensible allocation, especially when those containers or cargoes belong to different cargo owners? In such a situation, should we allocate the emissions using a voyage-based or leg-based method? For the partial return trip, where empty containers may also account for a

Table 3 Exemplary of input data

\begin{tabular}{lllc}
\hline $\begin{array}{l}\text { Combination of } \\
\text { weight and value }\end{array}$ & Inputs & & \\
\cline { 2 - 4 } & Quantity (TEU) & Unit weight (ton) & Unit value \\
\hline 1. Light-high & 150 & 15 & 1,000 \\
2. Light-low & 200 & 15 & 200 \\
3. Heavy-high & 250 & 20 & 600 \\
4. Heavy-low & 400 & 20 & 300 \\
\hline
\end{tabular}

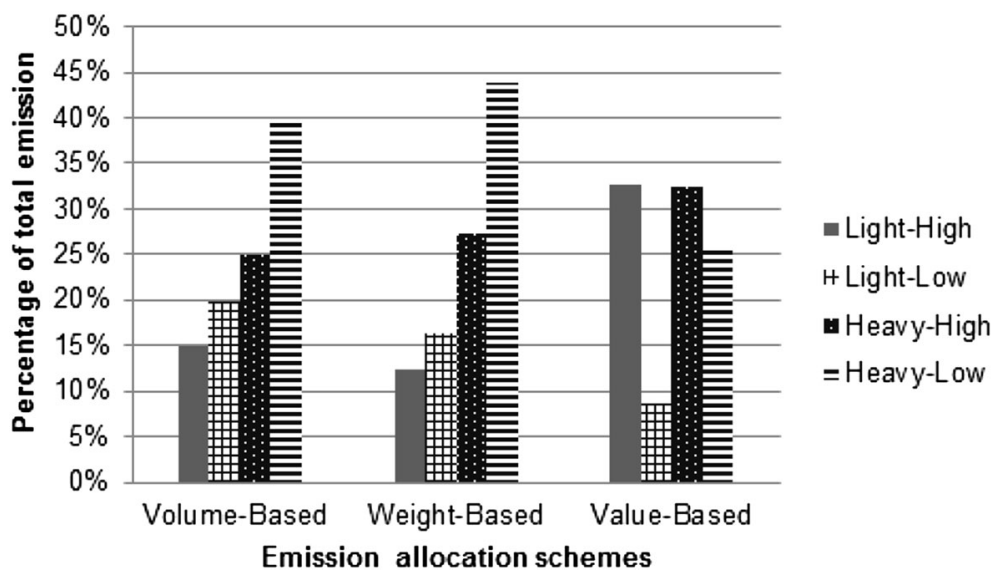

Fig. 4 Results of three different emission allocation approaches 
large portion of the total number of containers in a given trip, who should take care of the return cargo and/or empty containers? How much should they take? We discuss alternative emission allocation policies for container shipping in this section.

\section{Container shipping with empty return}

In this case, we have $m \in M=\{1,2\}=\{$ Heavy container, Light container $\}$, and $i, j \in N=\{A, B\},(i, j) \in A_{m}=\{A B, B A\}$. Then, $Q_{1 i j}$ refers to the number of heavy containers on leg $i j$ and $Q_{2 i j}$ is the number of light containers on leg $i j$.

In the first step, using the voyage-based method, we can calculate $P_{m}$ in the following way:

$$
P_{m}=\frac{\gamma_{m} Q_{m}}{\gamma_{1} Q_{1 A B}+\gamma_{2} Q_{2 A B}}, \quad m \in M=\{1,2\}
$$

According to the allocation scenarios presented in the above section, $\gamma_{m}$ could be decided based on the average weight, size (TEU), or freight rate of each type of container. The first option is based on the weight of each container. However, this method is not always suitable to represent the operation practices of container shipping because its weight is not the main restriction of capacity utilization in most cases. Another simple alternative could be volume-based (size-based) because this container is a type of standard package. We can assume that $\gamma_{m}$ for $40 \mathrm{ft}$ container is twice as that for $20 \mathrm{ft}$ container $\left(\gamma_{40} \mathrm{ft}=2 \gamma_{20} \mathrm{ft}\right)$. Moreover, if we choose economic value of each container, for example, the relevant data may not be so easily accessible. Therefore, the volume-based method is recommended for use in container shipping in most cases when allocating emissions to each container. We can obtain the emissions per container directly based on Eq. (4).

So far, this allocation is as simple as in the first case discussed and the only difference is that the cargo at this stage refers specifically to the container. In some cases, this type of allocation may be enough because the container is the actual handling and transportation unit. However, more detailed allocations may be helpful when the cargoes belong to different cargo owners. Some cargo owners need to know the emissions of one specific product for the entire life cycle, including transportation. Under such circumstances, the second step is needed.

In general, container cargo can be divided into different classes according to their suitability for container shipping and in terms of technological and economic feasibility. There can be various types of cargo, such as coffee, electronics, alcohol, pulp and cars. We can see that the difficult aspect in this case is that the cargoes in a container may have different characteristics. One method of approaching this allocation is based on the freight rates of different cargoes within each container. In general, a shipping company sets freight rate charges according to the following rules:

- "W"-based on gross weight

- "M"-based on volume

- "W/M" - based on the weight and volume (the higher one is chosen to use) 
Table 4 Input data with empty containers

\begin{tabular}{lllc}
\hline $\begin{array}{l}\text { Combination of } \\
\text { weight and value }\end{array}$ & Inputs & & \\
\cline { 2 - 4 } & Quantity (TEU) & Unit weight (ton) & Unit value \\
\hline 1. Light-high & 150 & 15 & 1,000 \\
2. Light-low & 200 & 15 & 200 \\
3. Heavy-high & 250 & 20 & 600 \\
4. Heavy-low & 400 & 20 & 300 \\
5. Empty & 400 & 2 & 10 \\
\hline
\end{tabular}

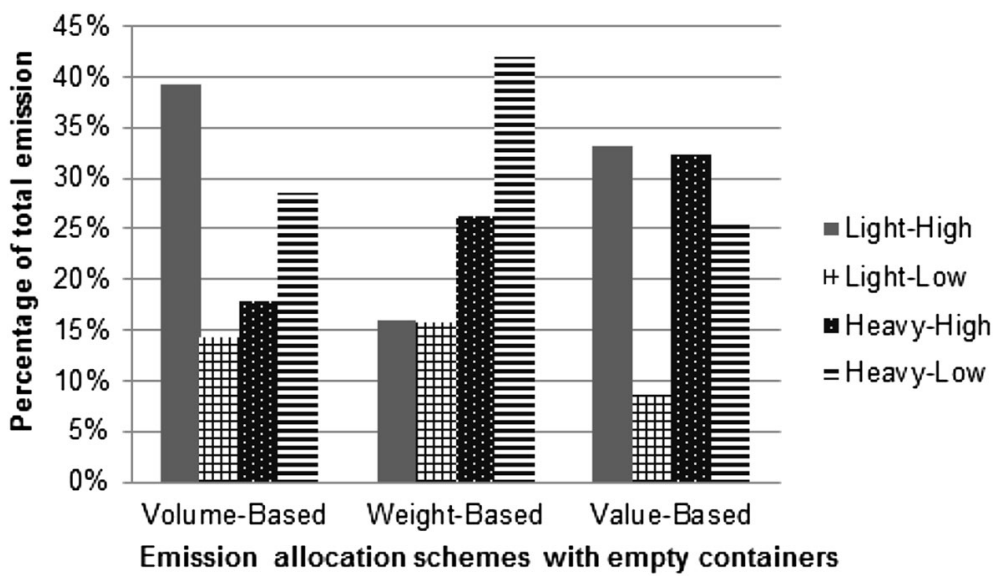

Fig. 5 Results of emission allocation with empty containers

- "Ad.Val." - based on FOB (Free on Board) price of the cargo

- "Ad.Val. or W/M" - based on FOB price, weight and volume (the highest one is chosen to use).

We therefore find that when a shipping company implements different freight rate charges for different cargoes, various cargo characteristics have already been taken into account. Hence, the freight rate could be used as a basis for calculating $\gamma_{m}$ in this step, representing a value-based approach.

Another important issue is how to allocate emissions from empty containers. According to the principle of Completeness described in the second section, all emissions should be allocated to the transported cargoes. Therefore, it is not sensible to allocate emissions directly to empty containers because there is no real cargo inside. We suggest allocating this portion of emissions to other non-empty containers on the same roundtrip. It is reasonable to figure out the real owner of these empty containers in this shipment period and allocate this part of emissions to the related cargoes. The same way of allocating emissions among other non-empty containers in this shipment can be applied.

We return to the example from the beginning of this section. We assume that there exist 400 TEU empty containers belonging to the same cargo owners of LightHigh containers during this shipment (see Table 4). Accordingly, the emissions 
from empty containers are allocated to these Light-High containers. The new result is presented in Fig. 5. We can find that an apparent change happens to the volume-based allocation scheme among these four types of non-empty containers because of the large number of empty containers. On the other hand, since the value of empty containers is limited, there is almost no change in the result based on the value-based scheme. It is noted that the reallocated results could be different if these emissions from empty containers are assigned to other non-empty containers.

\section{Container shipping with return goods}

In this section, we discuss the scenario of container shipping with return goods. The same assumption as above can still be applied in the example here.

For the voyage-based method, in which the emissions from the complete roundtrip are allocated and shared by each cargo type, $P_{m}$ can be obtained based on Eq. (2).

Accordingly, the unit emission for each type of container can be expressed as follows:

$$
e_{m}=\frac{E P_{m}}{Q_{m A B} D_{A B}+Q_{m B A} D_{B A}}, \quad m \in M=\{1,2\}
$$

For the leg-based method, the unit emission of each container can be obtained in the following way. The emission distribution is calculated as follows:

$$
\left(P_{m}\right)_{i j}= \begin{cases}\frac{\gamma_{m} Q_{m A B}}{\left(\gamma_{1} Q_{1 A B}+r_{2} Q_{2 A B}\right)}, & \text { if on leg AB } \\ \frac{\gamma_{m} Q_{m B A}}{\left(\gamma_{1} Q_{1 B A}+r_{2} Q_{2 B A}\right)}, & \text { if on leg BA }\end{cases}
$$

Here $\left(P_{m}\right)_{i j}$ refers to the allocation distribution for cargo $m$ on leg $i j$.

Then, the unit emission of each type of container on each leg is:

$$
\left(e_{m}\right)_{i j}=\frac{E_{i j}\left(P_{m}\right)_{i j}}{Q_{m i j} D_{i j}}, \quad m \in M=\{1,2\},(i, j) \in A_{m}=\{A B, B A\}
$$

Both approaches involve reasonable calculations and conformation to the Consistency, Completeness and No Redundancy principles. In this case, we should thus pay more attention to the principles of Individual Rationality and Motivation to determine which method is most helpful for the improvement and subsequent benchmarking. The cargo owners could be different for the forward and return routes. Their different requirements for cargo type, quantity, and timing vary greatly and have an impact on the choice of vessel speed, fuel type, cargo handling rates and other input parameters. This leads to different emissions on these two legs. Therefore, when we allocate the emissions to each container or to cargoes inside the container on each leg individually, we can search for better solutions to environmental efficiency improvement and, at the same time, satisfy the various requirements.

We provide a brief summary of these three allocation schemes in Table 5. 
Table 5 Summary of different allocation methods for illustrated examples

\begin{tabular}{|c|c|c|c|}
\hline & $\begin{array}{l}\text { Application } \\
\text { examples }\end{array}$ & Allocation schemes & $\begin{array}{c}\text { Remarks on application } \\
\text { examples }\end{array}$ \\
\hline 1 & $\begin{array}{l}\text { Single cargo- } \\
\text { type vessel } \\
\text { with empty } \\
\text { return }\end{array}$ & $\begin{array}{l}\text { All emissions from the complete roundtrip are } \\
\text { allocated to each cargo unit of the single type of } \\
\text { cargo transported on the forward trip. }\end{array}$ & $\begin{array}{l}\text { All emissions for a } \\
\text { complete trip, including } \\
\text { the empty return, should } \\
\text { be allocated to the } \\
\text { transported cargo. The } \\
\text { real-life cases can be } \\
\text { based on iron ore, coal, } \\
\text { and grain transport. }\end{array}$ \\
\hline 2 & $\begin{array}{l}\text { Single cargo- } \\
\text { type vessel } \\
\text { with another } \\
\text { return } \\
\text { cargo; } \\
\text { one type of } \\
\text { cargo is } \\
\text { involved in } \\
\text { the entire trip }\end{array}$ & $\begin{array}{l}\text { Alternative 1-A voyage-based method: } \\
\text { All emissions from the complete roundtrip are } \\
\text { allocated and shared with the total cargo } \\
\text { transported. } \\
\text { - Weight-based: The allocation is based on } \\
\text { the weight of each cargo; } \\
\text { - Volume-based: The allocation is based on } \\
\text { the capacity of the vessel used for each } \\
\text { cargo type; } \\
\text { Value-based: The allocation is based on the } \\
\text { economic value. } \\
\text { Alternative } 2-\text { A leg-based method: } \\
\text { Emissions are allocated among the forward and } \\
\text { return leg and are divided by their respective } \\
\text { transport work. We determine the unit emission for } \\
\text { each cargo on each leg separately. }\end{array}$ & $\begin{array}{l}\text { For this relatively simple } \\
\text { logistics chain, the unit } \\
\text { emission based on the } \\
\text { completed round trip } \\
\text { (Alternative 1) is the } \\
\text { preferred means of } \\
\text { allocation. }\end{array}$ \\
\hline 3 & $\begin{array}{l}\text { Partly uniform } \\
\text { cargo }\end{array}$ & $\begin{array}{l}\text { In this paper, we use container shipping as an } \\
\text { example. } \\
\text { Alternative } 1-\text { A voyage-based method: } \\
\text { In general, the emissions are allocated to each type } \\
\text { of container first. Then, the emissions of each } \\
\text { container can be allocated to the cargoes in the } \\
\text { container if necessary. } \\
\text { - Weight-based: The allocation is based on } \\
\text { the average weight of the full container and } \\
\text { the empty container; } \\
\text { - Volume-based: The allocation is based on } \\
\text { the container size ( } 20 \mathrm{ft}=1 \text { TEU and } 40 \mathrm{ft}= \\
2 \text { TEU); } \\
\text { Value-based: The allocation is based on the } \\
\text { economic value (for instance, freight rate) of } \\
\text { each container. } \\
\text { Alternative } 2-\mathrm{A} \text { leg-based method: } \\
\text { The allocation is accomplished in the same way as } \\
\text { performed for the entire trip. We generate the unit } \\
\text { emission for each type of container on each leg } \\
\text { separately. }\end{array}$ & $\begin{array}{l}\text { For a complex logistics } \\
\text { chain such as container } \\
\text { shipping, we can allocate } \\
\text { the emissions based on } \\
\text { each container or the } \\
\text { cargoes inside on each } \\
\text { individual leg (Alternative } \\
\text { 2). In this way, we can } \\
\text { search for the better } \\
\text { solutions to } \\
\text { environmental efficiency } \\
\text { improvement while } \\
\text { satisfying the various } \\
\text { proposed requirements. }\end{array}$ \\
\hline
\end{tabular}

\section{Conclusion and future work}

In this paper, we have developed a number of general principles to which such a scheme should adhere to determine the appropriateness of a certain allocation scheme for a given scenario. These principles include the Completeness, No Redundancy, Simplicity, Fairness, Individual Rationality, Motivation, and Consistency. We believe these principles are universal and are relevant not only for the 
allocation schemes discussed in this paper but also for the validation of a more complex, compound scheme for multimodal logistics chains.

We have also proposed alternative emission allocation schemes for maritime logistics chains. A systematic approach based on set of fundamental principles provides a clearer responsibility of carbon emissions and a stronger motivation for emission reduction. Furthermore, we have discussed alternative allocation schemes for different scenarios. These scenarios include a single type of cargo with empty return, a single type of cargo with return cargo, and partly uniform cargo, with and without return cargo. Based on the application examples for these three scenarios, we have concluded that the emissions from the empty return trip should be allocated to the cargoes on the forward trips. For the logistics chain with return cargoes, it is better to allocate the emissions based on averages in terms of environmental efficiency improvement. Moreover, for the scenario of partly uniform cargo (and especially container shipping), we have proposed a specific two-step process and recommended emission allocations to each leg individually since many input parameters impact the environmental efficiency of the container-shipping network.

The focus in this paper has been on the sea transportation leg of the logistics chain. Future work needs to extend this by addressing multimodal logistics chains. This will require the handling of complex multi-stage movements with numerous service providers and transportation modes. Additionally, allocation schemes relevant for the repositioning problem needs to be further developed. Finally, our contributions to the field of emission allocation should be combined with additional cost allocation schemes, thus providing a complete framework for the benchmarking and improvement of maritime logistics chains.

\section{References}

Asbjørnslett BE, Lindstad H, Mørkve OT (2010) Energy efficiency and environmental performance of maritime logistics chains: estimating the current situation-major challenges and possible pitfalls. In: 12th World Conference on Transport Research (12th WCTR), Lisbon, Portugal

Cadarso MA, López LA, Gómez N, Tobarra MA (2010) $\mathrm{CO}_{2}$ emissions of international freight transport and offshoring: measurement and allocation. Ecol Econ 69(8):1682-1694

Chakravarty S, Chikkatur A, de Coninck H, Pacala S, Socolow R, Tavoni M (2009) Sharing global $\mathrm{CO}_{2}$ emission reductions among one billion high emitters. Tech Rep, Princeton Environmental Institute, Princeton University, Princeton

den Elzen M, Olivier J, Berk M (2007) An analysis of options for including international aviation and marine emissions in a post-2012 climate mitigation regime. Tech Rep, Netherlands Environmental Assessment Agency (MNP)

den Elzen MG, Beltran AMJ, Hof AF, van Ruijven B, van Vliet J (2013) Reduction targets and abatement costs of developing countries resulting from global and developed countries reduction targets by 2050. Mitig Adapt Strateg Glob Change 18:491-512

Faber J, Markowska A, Nelissen D, Davidson M, Eyring S, Cionni I, Selstad E, Kgeson P, Lee D, Buhaug y, Lindtsad H, Roche P, Humpries E, Graichen J, Games M, Schwarz W (2009) Technical support for european action to reducing greenhouse gas emissions from international maritime transport. Tech Rep, CE Delft

Fet AM, Michelsen O, Johnsen T (2000) Environmental performance of transportation - a comparative study. Tech Rep, Norwegian University of Science and Technology 
Figliozzi M (2006) Analysis and evaluation of incentive-compatible dynamic mechanisms for carrier collaboration. Transp Res Rec: J Transp Res Board 1966:34-40

Frisk M, Gothe-Lundgren M, Jornsten K, Ronnqvist M (2010) Cost allocation in collaborative forest transportation. Eur J Oper Res 205(2):448-458

Groenenberg H, Blok K (2002) Benchmark-based emission allocation in a cap-and-trade system. Clim Policy 2(1):105-109

Hagemester C, Kristensen HOH (2011) Environmental performance evaluation of popax ferries. Ship Offshore 3:10-14

Heitmann N, Khalilian S (2011) Accounting for carbon dioxide emissions from international shipping: burden sharing under different unfccc allocation options and regime scenarios. Mar Policy 35(5):682-691

Houghtalen LM (2007) Designing allocation mechanisms for carrier alliances. PhD thesis, Georgia Institute of Technology

IMO (2000) Study of greenhouse gas emissions from ships. Tech Rep, Norwegian Marine Technology Research Institute-MARINTEK

Jackson T, Papathanasopoulou E, Bradley P, Druckman A (2006) Attributing UK carbon emissions to functional consumer needs: methodology and pilot results. Tech Rep, University of Surrey

Kontovas C, Psaraftis HN (2011) Reduction of emissions along the maritime intermodal container chain: operational models and policies. Marit Policy Manag 38(4):451-469

Krajewska MA (2007) Horizontal cooperation among freight carriers: request allocation and profit sharing. J Oper Res Soc 59(11):1483-1491

Leonardi J, McKinnon A, Palmer A (2010) Guidance on measuring and reporting greenhouse gas (GHG) emissions from freight transport operations. Tech Rep, The UK's Department for Transport (DfT)

LIPASTO (2009) Methodology for unit emissions of ships. http://lipasto.vtt.fi/yksikkopaastot/indexe.htm

MARLEN (2010) MARLEN methodology for calculating GEEC. Tech Rep, MARINTEK, DNV

McKinnon AC (2010) Product-level carbon auditing of supply chains: environmental imperative or wasteful distraction. Int J Phys Distrib Logist Manag 20(1/2):42-66

McKinnon AC, Cullinane S, Browne M, Whiteing A (2010) Green logistics: improving the environmental sustainability of logistics. Kogan Page, London

Naber S (2012) Allocation $\mathrm{CO}_{2}$ emissions to customers on a distribution route. Master's thesis, Erasmus University Rotterdam

Olson EG (2010) Challenges and opportunities from greenhouse gas emissions reporting and independent auditing. Manag Audit J 25(9):934-942

Rajagopal D, Zilberman D (2010) On environmental lifecycle assessment for policy selection. Tech Rep, University of California. http://escholarship.org/uc/item/39k9h976

Sagar AD (2000) Wealth, responsibility, and equity: exploring an allocation framework for global ghg emissions. Clim Change45(3):511-527

Sandvik ET (2005) Environmental impacts of intermodal freight transport. Tech Rep, Molde University College

SBSTA (1996) National communication by the subsidiary body for scientific and technological advice, fccc/sbsta/1996/9/add.1. Tech Rep

Sijm J, Jansen J, Torvanger A (2001) Differentiation of mitigation commitments: the multi-sector convergence approach. Clim Policy 1(4):481-497

UNFCCC (2003) UNFCCC (2003) caring for climate: a guide to the climate change convention and the kyoto protocol. Tech Rep, the Climate Change Secretariat UNFCCC Bonn, Germany

Vrenken H (2005) Intermodal transport in Europe. European Intermodal Association

Wick C, Klumpp M, Kandel C (2011) Shapley value simulation for allocating GHG emission savings due to logistics pooling within ECR cooperations. In: Novais P, Machado J, Analide C, Abelha A (eds) The 2011 European Simulation and Modelling Conference, University of Mino, Guimaraes. The European Technology Institute Bvba (ETI Bvba), Ostend, pp 240-244

Wood FR, Bows A, Anderson K (2008) How can $\mathrm{CO}_{2}$ emission from aviation be allocated to reginal administrations fro monitoring and target setting. Tech Rep, Tyndall Centre, University of Manchester

Ziegler F, Hansson PA (2003) Emissions from fuel combustion in Swedish cod fishery. J Clean Prod 11(3):303-314 